\title{
Edificación de la competencia motriz y conquista de las realizaciones personales
}

\author{
The building of motor competence and the conquest of personal fulfillments
}

Luis Felipe Brito Soto*

\section{Resumen}

Palabras clave: Crecimiento y desarrollo, modelos motrices, desarrollo de competencias, capacidades físico-motrices.

\section{Abstract}

Key Words: $\quad$ Growth and development, motor function models, competence development, physical/motor skills.

Fecha de recepción: 22 de agosto de 2006

Fecha de aceptación: 26 de octubre de 2006

1 Universidad Pedagógica Nacional de México. Subsecretario en la Secretaría de Educación Pública de México. 


\section{Introducción}

Actualmente la educación física cuenta con mejores explicaciones y planteamientos pedagógicos para comprender el significado que tienen las acciones motrices de niños y adolescentes en el ámbito escolar.

Estas explicaciones han sido configuradas desde muchas perspectivas y con distintos propósitos. De ellas, la de mayor auge es la que mira el crecimiento y desarrollo de niños y adolescentes dividido en fases, con énfasis en el rendimiento del cuerpo y la estimulación de las capacidades motrices básicas. Hoy podemos pensar en forma distinta, pero, sobre todo, explicar nuestra intervención docente para proporcionar actividades valiosas a los escolares desde la orientación de la acción motriz -que considera relevante solucionar problemas- y el enfoque dirigido a la relación entre los procesos de pensamiento y los desempeños motrices que hacen de éstos últimos actos inteligentes.

El debate conceptual acerca del desarrollo motor ha evolucionado. Sabemos que la motricidad encierra relaciones entre el movimiento y los procesos cognitivos y afectivos, que existe interacción tanto con el medio como con la comunicación con los demás: que las respuestas y desempeños motrices van perfeccionándose a partir de una percepción clara de los propósitos de la acción misma; que pueden realizarse con más sentido y con mejor desempeño los cometidos si conocemos la lógica y la dinámica de las actividades y, sobre todo, que es importante poseer una aptitud para combinar los modelos motrices internos con los externos, con el fin de obtener una motricidad variada, original y novedosa (Castañer y Camerino, 1991). Cierto es también que los distintos enfoques conviven y se tocan constantemente para proporcionar a los profesionales de este campo elementos para tomar decisiones en torno al qué y al cómo del quehacer de la educación física que se brindan a los escolares.

Ante esta situación, se hace necesario debatir y contrastar nociones y explicaciones acerca del aporte de la educación física a los escolares. En este sentido, apostamos por el desarrollo de competencias, orientación que abarca mucho más las realizaciones de niños y adolescentes que la visión del rendimiento. Así, es pertinente considerar el desarrollo motor como un proceso dinámico en la adquisición y edificación de la competencia motriz, característica central de la enseñanza de la educación física para impulsar en los escolares una interacción eficaz con el medio.

Una competencia es una adquisición, una construcción personal, y no necesariamente un producto de la carga genética de los individuos. Ser competente significa aprender a identificar nuestras propias potencialidades y aceptar nuestros límites al momento de resolver una tarea o cometido. ¿Podemos contribuir a su construcción? ¿Las habilidades motrices pueden ser consideradas como competencias? ¿Qué propósitos educativos persigue la automatización de un movimiento? ¿Los niños no están cansados de saltar dentro y fuera del aro? ¿Mejoran las pruebas del rendimiento físico y sus resultados, la propuesta curricular y las capacidades físicomotrices? La reflexión que se presenta a continuación trata de debatir sobre éstas y otras interrogantes. En el centro de la discusión está la competencia motriz como eje de enseñanza de la educación física y, con ello, la perspectiva pedagógica de las sesiones de educación física, donde niños y adolescentes logren conquistas personales e incorporen a su personalidad importantes aprendizajes desde la acción motriz

\section{La educación física, una intervención pedagógica y práctica educativa}

La intención pedagógica de la educación física vincula los desempeños motrices de las personas con sus motivaciones e intereses, sus capacidades y limitaciones. Por su parte, la práctica educativa activa y motiva los tres mecanismos efectores del movimiento: sensaciónpercepción, formulación de planes de acción y toma de decisiones, y ejecución.

Al efectuar una acción motriz se movilizan y activan innumerables procesos que permiten a niños, niñas y adolescentes imaginar cómo realizar determinadas tareas o acciones motrices, para después llevarlas a cabo. La sensación-percepción proporciona la primera idea, la primera imagen mental, el esbozo general, la representación anticipada de lo que hay que realizar. En este momento el sujeto percibe el grado de dificultad que implica la tarea que le ha sido asignada, se percata del esfuerzo que debe invertir en su solución; antes de lanzarse a realizar el cometido en cuestión, toma una decisión sobre la cantidad de esfuerzo que requiere e imagina cómo puede hacerlo. A este proceso se le llama preactivar.

La intervención docente provoca, por medio de distintas vías, la primera idea de ejecución. El maestro, a través de verbalizaciones, movimientos, uso de objetos o ejemplificación a través de alumnos, proporciona la estructura general y la dificultad de la tarea. Por su parte, los niños contrastan entre la tarea solicitada y la percepción de su propia competencia: ¿Podré hacerlo?... 
Maestro, eso es muy fácil! ¿Y si to hacemos mejor así? La personalidad de los sujetos y la percepción que tienen de su propia competencia influyen en la elección del grado de esfuerzo por realizar (Famose, s. f.).

Durante este primer momento (sensación-percepción), es muy importante dar paso a la experimentación continua de situaciones motrices a través del ensayo y el error. Como se ha señalado, esto permite a niños, niñas y adolescentes preactivar soluciones a los diversos problemas cinéticos que enfrentan.

Aquí hay un elemento muy importante para una adecuada intervención docente. Si dentro de las sesiones de Educación Física las tareas y cometidos motrices presentan una dificultad acorde con las características de crecimiento y desarrollo de los estudiantes, y si sus intentos y realizaciones son satisfactorios, ellos se perciben como competentes. En consecuencia, la percepción de la tarea y su realización exitosa les procura un sentimiento de eficacia, un placer interno y una alegría; incluso, es probable que vuelvan a intentarlo por su cuenta (iniciativa propia)

Muchos autores (Jean-Pierre Famose, s. f.; Pierre Parlebas, 1995, Jean Le Boulch, 1976; Pierre Vayer, 1977 y B. Cratty, 1982) dan cuenta de este proceso. Se movilizan las experiencias previas, la memoria a corto y mediano plazos, la audición, la observación y la toma de conciencia de toda la estructura propioceptiva del cuerpo. Durante este desarrollo, los niños están contrastando su propia movilización motriz a partir de las consignas que van mencionándose, así como por los cambios que se producen dentro del contexto de la sesión.

\section{Ejemplo}

El profesor propone realizar una actividad dentro de una zona circular y solicita desplazamientos cortos y rápidos (aquí determina las características y la dificultad de la tarea). Posteriormente, introduce una pelota y activa un juego de pases (cita niveles más complejos de los desempeños). Si el jugador no atrapa la pelota, se quema y queda fuera del círculo descansando durante un minuto.

Esta secuencia, primero es explicada por el profesor (antes de la acción misma) y luego se pone en marcha. Durante la explicación los niños hacen representaciones mentales de la tarea, anticipan posibles respuestas y toman decisiones para configurar un plan de acción de movimiento personal.
Durante la puesta en marcha -donde se ubican los mecanismos de formulación de planes de acción, la toma de decisiones y la propia ejecución-, se modifican los planes de acción originales, se ajustan y se realizan movimientos de acuerdo con las exigencias de la actividad y la sucesión de acciones. En este proceso se presentan muchas posibilidades pero, sobre todo, se construye el conocimiento relacionado con el saber hacer. Es decir, se han puesto a prueba las habilidades motrices, que pueden conceptuarse como:

La competencia adquirida por un sujeto para realizar una tarea concreta. Se trata de la capacidad para resolver un problema motor específico, para elaborar y dar respuesta eficiente y económica, con la finalidad de alcanzar un objetivo preciso. Es el resultado de un aprendizaje, a menudo largo, que depende del conjunto de recursos de que dispone el individuo, es decir, de sus capacidades para transformar su repertorio de respuestas (Duránd, 1999).

Ahora bien, este repertorio de respuestas - los mecanismos efectores del movimiento ya mencionados- se fusionan con el sentimiento de ser competente, que puede desglosarse en tres ámbitos: a) el cognitivo, que se refiere a la capacidad del individuo para imaginar respuestas motrices - pues comprende el problema, la dificultad de la tarea, y hace una primera elaboración de respuesta-. b) El afectivo, que tiene que ver con la atmósfera de confianza que el ambiente le ofrece (grupo de amigos, cordialidad, seguridad). c) El físico, que atañe al conocimiento de las posibilidades corporales y motrices para efectuar la tarea en cuestión. En este contexto, la habilidad motriz está marcada por su carácter personal, flexible, en construcción, eficiente, estable y adaptable. La pertinencia del ejemplo anterior radica en que los niños y las niñas logran una experiencia estimulante y satisfactoria; confían en sus propios movimientos y realizaciones; logran una experiencia y escogen ser activos, seguros y probablemente persistirán en la búsqueda variada y novedosa de formas de ejecución.

Entonces, la acción y la respuesta motriz que los niños hacen a partir de la percepción de la tarea.

va más allá de la ordenación pasiva de lo recibido del exterior, mediante la cual determina, filtra y pone condiciones a esa estimulación externa; representando el aprendizaje y la interacción con otros procesos cognitivos un papel fundamental. No recibimos, por tanto, pasivamente los estímulos del medio, sino que decidimos cuál de ellos seleccionamos y lo organizamos en interacción con los procesos de memoria, atención y programación (Famose, s. f.). 
Volviendo al punto, la intervención docente vincula los mecanismos efectores del movimiento con las expectativas e intereses de los niños y adolescentes $y$, sobre todo, promueve la construcción de competencias para solucionar problemas motrices dentro de las sesiones de educación física.

La intervención docente forma en competencias, impulsa la adquisición de experiencias, anima la construcción de aprendizajes, y en educación física trabaja más allá de lo que se ha considerado el potencial anatómico.

Formar en competencias y formar la competencia motriz equivale -entre otros factores- a motivar a los niños, las niñas y los adolescentes para que identifiquen y encuentren los conocimientos y experiencias adecua dos y las posibles realizaciones motrices, tenerlas vigentes, organizarlas y designarlas dentro de un contexto. La acción motriz inteligente y la edificación de la competencia motriz pasan por razonamientos, toma de decisiones conscientes, tanteos, dudas, ensayos y errores. El desafío, desde esta perspectiva, es potenciar el aprendizaje de los alumnos y dirigirlo a construir su sentimiento de ser competente, de sentirse apto.

\section{Educación física y competencia motriz}

Una persona es competente cuando actualiza su conocimiento de saber cómo hacer las cosas: este tipo de conocimiento tiene un carácter práctico y

...se interesa por ciertas formas de competencia

activa, como cuando digo: "sé conducir un coche",

"sé hacer una estantería" o "sé jugar al tenis"... se

interesa por la ejecución de propósitos de acción,

efectuados de una manera racional y confirmados

por un grado razonable de éxito (Carr, citado por

Arnold, 1997). Lo más sobresaliente aquí es que, cuando una persona "sabe cómo hacer" ciertas cosas específicas (por ejemplo, una mesa o jugar al squash), su conocimiento se ha actualizado y puesto en práctica en lo que hace. Su inteligencia se manifiesta en sus hechos, destrezas y realizaciones (Arnold, 1997).

Este tipo de conocimiento permite a las personas resolver situaciones nuevas y diferentes.

El saber cómo no implica solamente las realizaciones de corte práctico. Se fusiona además con la posibilidad de las personas para argumentar sus acciones. A esto, Arnold le llama sentido débil y sentido fuerte del saber cómo. El sentido débil se refiere a la persona que es incapaz de explicar cómo hizo las cosas, mientras que el sentido fuerte alude a una persona que puede propor cionar una relación descriptiva, clara y penetrante de cómo lo hizo. "Pregunten al brillante, intuitivo, pero inexpresivo portero, cómo evitó un gol. Puede que diga 'fue así y trate de mostrarlo, en vez de explicar cómo' (Arnold, 1997).

Ahora bien, este saber cómo, si lo relacionamos con los desempeños motrices y la disponibilidad corporal desplegados en las sesiones de educación física, vigoriza una constante interacción con el medio, ya que genera respuestas motrices que permiten salir airosos pero, sobre todo, reconocer el peso de la inteligencia sobre las acciones.

A esta disponibilidad Ruiz Pérez (1995) le llama competencia motriz: conjunto de conocimientos, procedimientos, actitudes y sentimientos que intervienen en las múltiples interacciones que realiza en su medio y con los demás, y que permiten que los escolares superen los diferentes problemas motrices planteados, tanto en las sesiones de Educación Física como en su vida cotidiana.

La competencia motriz y el tipo de conocimientos que despliega vigorizan la disponibilidad corporal de los alumnos y contribuye a tener juicios propios al momento de tomar decisiones de cómo hacer las cosas. Esta competencia implica "conocer qué hacer, cómo hacerlo, cuándo y con quién actuar, en función de las condiciones cambiantes del medio" (Arnold, 1997), tener dominio, desempeñarse con seguridad y apropiarse de distintos saberes en relación con la propia acción y la de los demás proporcionadas por el contexto.

En la edificación y desarrollo de la competencia motriz participan tres tipos de conocimiento: declarativo procedimental y estratégico. La conjugación de estos saberes constituye la adquisición de una competencia, conocimiento que implica mayormente un carácter procedimental, y que se traduce en el 'saber cómo'. Éste a su vez se expresa en: saber hacer, saber actuar y saber desempeñarse.

\section{El saber hacer: vínculo entre el conocimiento procedimental y la destreza motriz}

El saber hacer -declarativo- es la primera conexión con el saber cómo, y se refiere básicamente a la acumula ción perceptiva y "al saber que los sujetos poseen sobre sus acciones y sobre los hechos y acontecimientos que rodean a su actuación" (Arnold, 1997), a los conceptos acerca de cómo se hacen las cosas y, en el tema que nos ocupa, a cómo son la lógica, la secuencia, la estructura, el tipo y la cantidad de desempeños motrices que exigen los juegos y actividades desarrolladas en las sesiones de 
Educación Física. En principio, esos componentes se incorporan al repertorio motriz de niñas y niños a través de las declaraciones, nociones e instrucciones que los adultos les proporcionamos en torno a lo que sucederá y cómo serán las acciones por desarrollar. Los profesores damos muchas indicaciones y datos con relación a la forma que deben tener los distintos desempeños y acciones corporales. Proporcionamos conocimientos declarativos mediante consignas que dan cuerpo a las destrezas, como: “...hemos de correr entre los obstáculos", “...las pelotas serán lanzadas hacia arriba y las atraparemos con los conos", “...las capturas se deberán hacer al toque de la espalda del compañero", etcétera.

Este tipo de informaciones constituye la base para edificar el saber hacer. Un ejemplo que nos permite clarificar este argumento es ver cómo los niños conocen y comprenden la lógica y la estructura interna de distintos juegos. Veamos el caso del beisbasquet ${ }^{1}$.

Un niño comprende la continuidad, la dinámica, la estructura y la lógica de la actividad, previamente a la realización de la acción misma y posee el conocimiento declarativo de este juego. Sabe, entre muchos otros conceptos e informaciones, que:

- En el equipo de beisbasquet somos nueve jugadores.

- Uno de ellos es el lanzador.

- La pelota debe rodar para que el jugador pueda pa tearla.

- Los demás debemos completar tres pases antes de quemar al jugador que corre.

- Se anotan las carreras que el equipo va logrando.

- Al quemar a tres contrarios, nosotros pasamos a patear la pelota...

Estos enunciados indican cómo han de realizarse los cometidos motrices (por ejemplo: las pelotas las atraparemos en los conos) y proporcionan el sentido y el significado de la motricidad. De ahí que el saber hacer es el paso previo a la acción.

Paralelamente, el conjunto de conocimientos declarativos proporcionan a los escolares toda

\footnotetext{
Este juego consiste en patear una pelota y correr en torno a tres bases diseminadas en un área rectangular. Los jugadores de campo, al tener $\mathrm{l}$ pelota, hacen tres pases entre ellos para después embocarla en una caja de cartón. Si toda esta acción se efectúa antes que el corredor recorra las base éste se "quema" y no contabiliza su carrera. Si, por el contrario, el corredor es más veloz que la secuencia de los tres pases, se contabiliza su recorrido. Se intercambian los roles de participación en el momento de "quemar" tres jugadores. Una "entrada" está compuesta por la participción de bo dos equipos en el momento que complat los tres que de
}

una serie de datos sobre hechos y acontecimientos relacionados consigo mismos, con su propio cuerpo, sobre la forma de sus acciones, de los objetos y sobre las relaciones espacio-temporales que afectan a las acciones en contextos muy diferentes (Ruiz, 1995).

Este cúmulo de conocimientos también posibilita a los niños y niñas

aprender el nombre de sus partes corporales y aprender a emplear este conocimiento declarativo para tomar decisiones, con la intención de conseguir los objetivos previstos; en definitiva, el conocimiento declarativo es un elemento necesario para el empleo de los conocimientos procedimentales conceptualizándose en redes semánticas (Ruiz, 1995).

Otro puente importante entre este tipo de saber y los cometidos motrices lo constituyen las reglas (ya de los juegos, ya de las actividades) y su conocimiento. Éstas dan el soporte a la dinámica y la gramática de la actividad, y cumplen y canalizan el orden de las acciones. $\mathrm{La}$ información proporcionada por las reglas y las declaraciones acerca de cómo son las realizaciones motrices dentro del juego y en qué consiste se ordena y codifica en el pensamiento para ser comprendida por los jugadores y poder conocer las posibilidades de acción, los límites de la actividad, la lógica de los desempeños, el móvil del juego y la forma de manipular el implemento. Todo ello les confiere a los juegos y a las actividades dos componentes básicos: medio y sistema. Como medio, posibilitan a un grupo de niños y niñas interactuar, relacionarse y trabajar juntos a partir de un interés y motivación común, y como sistema, constituyen y representan un acuerdo de realización y disfrute mutuo. Todo esto forma una buena parte del conocimiento del saber hacer.

\section{El saber actuar incorpora al yo en la acción}

Siguiendo con el ejemplo anterior de cómo es el jue go de beisbasquet, llega el momento de ponerse en acción, de jugar, de enfrentar ese saber hacer con los propios desempeños y la disponibilidad corporal. Al momento de jugar, el niño hace una serie de contrastaciones entre aquella idea perceptivo-motriz y el conocimiento procedimental que implica, con la acción concreta, ... ahora se trata de patear la pelota y correr lo más rápido posible antes de que me quemen...

Se pone en juego el conocimiento declarativo tanto de las realizaciones motrices como de las reglas, y la 
motricidad en conjunto se supedita a ellas, de tal manera que al ser el escenario de las posibilidades y desempeños motrices, requieren el ajuste y dominio de sí, la incorporación de toda la entidad corporal, sus emociones, sus ideas, sus sentimientos y, sobre todo, la comprensión cabal de la lógica y continuidad de la actividad. Estamos frente al aprendizaje de procedimientos.

Trabajar los procedimientos significa, en definitiva, desvelar la capacidad de saber hacer, de saber actuar de manera eficaz. Un procedimiento, señala Coll (1992), trata siempre de determinadas y concretas formas de actuar, cuya principal característica es que no se realizan de forma desordenada o arbitraria, sino de manera sistemática y ordenada, unos pasos detrás de otros (...) hacia la consecución de una meta.

Consiste en saber operar con objetos y con información. Una característica del aprendizaje y enseñanza de procedimientos es poner más énfasis en los procesos que en lo producido, más en cómo van haciéndose las tareas y cometidos que en los resultados finales.

Este tipo de conocimiento procedimental se centra en la relación que existe entre los aspectos perceptivos y cognitivos, con el control y dominio de las respuestas motrices y, como dice Ruiz, relacionado con el "cómo hacer algo", con la realización de una acción, con los procedimientos para llevar a cabo una secuencia motora y con los esquemas motrices que controlan la ejecución de las acciones.

\section{Saber desempeñarse: el toque estratégico}

El conjunto de conocimientos referidos a las reglas, sus posibles modificaciones, el aumento de complejidad de los juegos, la codificación y comprensión de las participaciones individuales y del grupo, el móvil y la aceptación de actitudes como la tolerancia y la serenidad ante el triunfo y la derrota forman parte, en alto porcentaje, del saber desempeñarse. Es decir, son el epicentro y la cristalización del nivel más alto de la motricidad ante el placer de jugar y al momento de realizar los cometidos motrices. En conjunto, agrupan el desarrollo de la competencia motriz.

En efecto, la suma del saber hacer y del saber actuar forman el soporte de la motricidad del desempeño motriz inteligente. Aquí yacen, enfatizando, el conocimiento, la comprensión y la actitud atenta para saber cómo se hacen los propios desempeños, pero, sobre todo, ir elaborando una solución -actuación estratégica- que no se tenía, lo que permite solucionar determinado problema para el cual no había solución de antemano. Lo estratégico consiste en la elaboración de un procedimiento, de un plan de acción personal, del cual se desprende el reconocimiento de sus límites y posibilidades.

Para el caso del desarrollo de la competencia motriz, es básico que los escolares posean un conocimiento sobre las propias acciones que les permita abonar la actuación estratégica, es decir, poseer un conocimiento procedimental, declarativo y afectivo relacionado con las acciones motrices (sentido fuerte del movimiento del que se comentó anteriormente), de tal manera "que los niños y niñas vayan tomando conciencia de lo que conocen sobre las acciones y cómo aprenden a realizar valoraciones sobre sus propias competencias" (Ruiz, 1995).

Con respecto a la comprensión de las realizaciones, individuales y de conjunto, dentro de los juegos y las actividades que despliega la Educación Física, vale la pena destacar cómo el contexto donde se desarrolla la acción y el nivel de incertidumbre que se presenta en muchos juegos provocan en los participantes una toma de decisiones y respuestas flexibles para adaptar e improvisar el juego. Aparecen entonces la solución a los cometidos motrices supeditados por las reglas, las respuestas a los desafíos planteados por la secuencia, el orden y la fluidez de las acciones por superar, las fintas, los cambios de dirección, la aceleración y frenado, y el manejo de los espacios de los adversarios; en síntesis, la actualización del conocimiento del saber desempeñarse decidiendo qué hacer, y cuándo y cómo realizarlo.

\section{Caminos para la intervención docente}

\section{La solución de problemas}

La intervención docente tiene varios retos: trabajar regularmente en la solución de problemas; crear o utilizar otros medios de enseñanza; negociar y conducir proyectos con los alumnos; adoptar una planificación flexible e indicativa, improvisar (Perrenoud, 1999). Para efectos del presente artículo, es conveniente ahondar en lo referente a trabajar regularmente a través de problemas.

Formar en competencias, dice Perrenoud (1999), exige una pequeña "revolución cultural", "las competencias se crean frente a situaciones que son complejas desde el principio”. ¿Cómo hacerlo desde la Educación Física? ¿La clase modelo forma en competencias? ¿Da paso a la diversidad y a la toma de decisiones por parte de los alumnos? ¿Enfrenta a los niños, las niñas y los adolescentes a la movilización de saberes y al uso de experiencias previas? 
Para edificar la competencia motriz debe ponerse a los estudiantes en situaciones que los lleven a alcanzar objetivos, resolver problemas y tomar decisiones (recordemos cuál es el punto nodal del mecanismo efector del movimiento). Por principio es conveniente recurrir a diversos tipos de situaciones-problema, unas con intención bien definida y otras que vayan surgiendo del avance del grupo. Para ello, podemos recordar el planteamiento sobre el deporte educativo de Le Boulch (1991), quien propone que una situación-problema

...posibilita el enriquecimiento del bagaje de automatismos disponibles para el ajuste. Pero una situación-problema es también punto de referencia, en la medida que permite hacer hincapié en el nivel de desarrollo del conjunto de las funciones psicomotrices y, secundariamente, de determinados factores de ejecución" (Le Boulch, 1991: 61).

¿Cómo aprovechar las situaciones-problema? Los propósitos por lograr mediante este tipo de situaciones resultan ser:

Experiencias favorables para encauzar actitudes sociales, de organización, comunicación y cooperación como componentes de la competencia motriz en tanto que se traduce en:

- Una conciencia más clara de la tarea que debe realizar el grupo y de las reglas de su funcionamiento.

- Una mejor aptitud para analizar los resultados obtenidos y para criticar la no adaptación del grupo a su tarea.

- Una mejor comprensión de los puntos de vista de los otros y la posibilidad de ubicarlos con relación a la propia opinión.

- Una participación activa en la vida grupal, aportando una cooperación creativa; por ejemplo, interviniendo para modificar las reglas o para proponer una determinada modalidad de acción (Le Boulch 1999: 59).

Ejemplos:

\begin{tabular}{|c|c|}
\hline Stuacibn real & Postble problemathacilon \\
\hline Adaptar el área de juego & ¿Y si lo hacemos en una cancha distinta? \\
\hline $\begin{array}{l}\text { Modificar cómo hacer un tanto } \\
\text { (cada tres cuenta como uno) }\end{array}$ & Inventemos formas de hacer anotaciones \\
\hline Replantear el reglamento & ¿Qué hacer para participar en grupo? \\
\hline $\begin{array}{l}\text { El que anota, pasa al equipo } \\
\text { contrario }\end{array}$ & ¿Cómo podemos todos hacer anotaciones? \\
\hline
\end{tabular}

Una situación-problema se caracteriza por seguir una secuencia didáctica formada por cuatro pasos:

\begin{tabular}{|c|c|c|c|}
\hline Paso 1 & Paso 2 & Pasto 8 & paso 4 \\
\hline $\begin{array}{l}\text { - Se expone el tema } \\
\text { en cuestión y sus } \\
\text { puntos de interés. } \\
\text { - Se motiva a los } \\
\text { alumnos para } \\
\text { iniciar la búsqueda } \\
\text { del problema en } \\
\text { cuestión. }\end{array}$ & $\begin{array}{l}\text { Se plantea } \\
\text { el propósito } \\
\text { a lograr } \\
\text { durante el } \\
\text { desarro- } \\
\text { llo de la } \\
\text { sesión. }\end{array}$ & $\begin{array}{l}\text { - Se proporciona la } \\
\text { información y los } \\
\text { datos necesarios } \\
\text { para canalizar } \\
\text { la búsqueda. } \\
\text { de soluciones } \\
\text { al problema y } \\
\text { cometido plan- } \\
\text { teado. }\end{array}$ & $\begin{array}{l}\text { - Se dan a cono- } \\
\text { cer las normas, } \\
\text { condiciones y } \\
\text { límites en los } \\
\text { que puede } \\
\text { desenvolverse } \\
\text { el problema. }\end{array}$ \\
\hline
\end{tabular}

Para definir qué puede ser un problema y las posibles formas de motivar su descubrimiento, hablaremos de tres ámbitos: el contexto del juego, sus exigencias, y los problemas y sus causas.

El contexto del juego: es el marco general donde se realizan los desempeños. Desde los materiales y áreas, hasta las relaciones e interacciones de los participantes y los propósitos de la misma acción. La siguiente gráfica nos permite clasificar esta idea (gráfica 1):

Una actividad o juego bien elegidos:

a) ¿Es motivante?

( No hay juego si no hay riesgo de perder y posibilidad de ganar).

b) ¿Permite una cantidad de trabajo suficiente?

- Problemas de la espera.

- Problemas de la eliminación.

- Problemas de los desplazamientos.

c) ¿Permite una mejora cualitativa de las acciones motrices?

- La motricidad no debe ser estereotipada.

- Los alumnos deben tomar decisiones.

- Mejorar las relaciones sociales.

Gráfica 1. Contexto de juego.

Los problemas pueden ser distintos y a partir de cualesquiera de ellos es posible confeccionar una situación específica. Por ejemplo, si tomamos el inciso b), en relación con el subsistema, podríamos proponer: problemas de la espera: ¿Cómo modificar la actividad para no tener a muchos compañeros esperando su turno de participación?

Sus exigencias: los requerimientos pueden ser de varios tipos, según la lógica propia de la actividad. No es lo mismo un juego de capturas (donde se tiene que correr, virar y esquivar, tocar al compañero, llegar a zonas 
neutrales), que un juego de blanco y diana (por ejemplo, tirar a los bolos desde una posición fija). La gráfica 2 nos permite ubicar el tipo de solicitudes de la acción elegida:

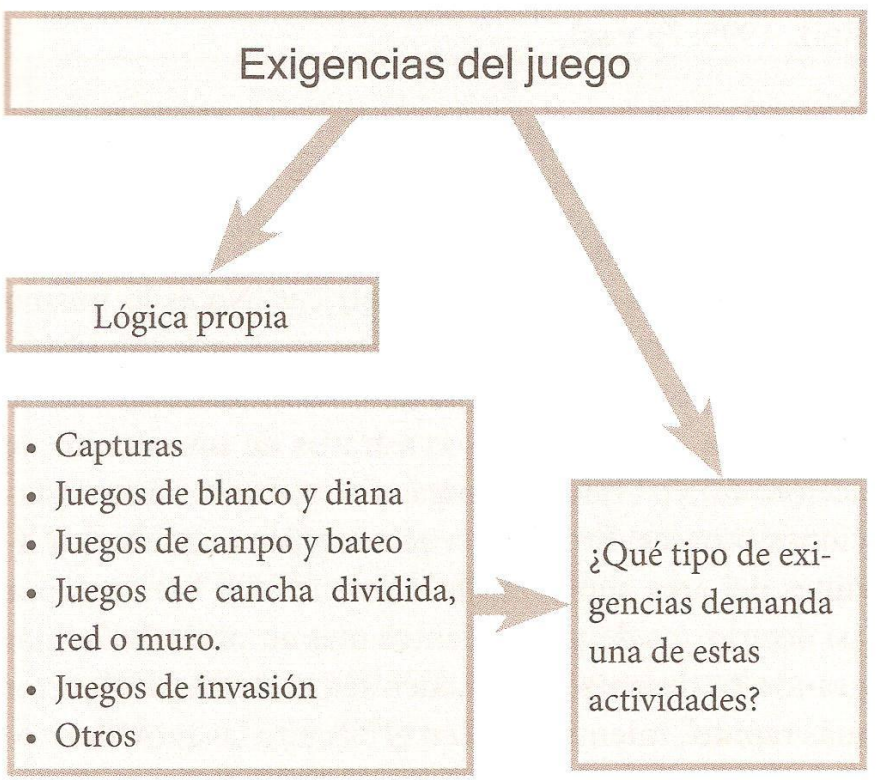

Grálica 2. Exigencias del juego.

Los problemas y sus causas: Momento durante el cual ubicamos los principales retos que el grupo enfrenta y se determinan las causas y sus posibles soluciones. El esquema respectivo es el siguiente (gráfica 3).
Problemas

$$
\begin{aligned}
& \text { Individuales } \\
& \text { - Lanzar, atrapar, parar, } \\
& \text { cambiar de dirección. } \\
& \text { - Situarse, orientarse. } \\
& \text { - Arrancar, pasar, actuar. } \\
& \text { Colectivos } \\
& \text { - Juego no comprendido. } \\
& \text { - Demasiado agrupados. } \\
& \text { - Acciones no coordinadas } \\
& \text { disputas. } \\
& \text { - Jugadores que se retiran. }
\end{aligned}
$$

\section{Colectivos}

Causas

- Malas percepciones (función de ajuste).

- Relaciones mal establecidas.

- Causas afectivas: miedo, indiferencia, al profesor, a la actividad misma o al material.

- Factores de ejecución: coordinación motriz general o de capacidades motrices.
Gráfica 3. Problemas y elección de soluciones.

Una situación-problema, como puede advertirse, no es una situación didáctica sin importancia. Por el contrario, exige del docente una inventiva para diseñar y construir ambientes y tareas que enfrenten a los alumnos a la movilización de saberes y experiencias. Renovación y variación didácticas deben ser los ejes rectores para activar la motricidad de niños y adolescentes de manera estimulante y sorprendente.

Dirigirse a la edificación de la competencia motriz obliga al docente a replantear su labor, ponerse en el lugar de los estudiantes, comprender lo que inhibe y lo que vigoriza su expresión motriz. Siguiendo a Perrenoud (1999: 78), ¿Esto supone una fuerte capacidad de comunicarse con el alumno, de ayudarlo a verbalizar lo que le turba o lo bloquea, de incitarlo a una forma de metacognición?

\section{Los patrones de movimiento y la variabilidad en la práctica}

Para activar una competencia es necesario aplicar cometidos motrices y situaciones-problema que contengan una variabilidad de la práctica. Ésta se sostiene porque:

- Una vez resuelto un problema -por ejemplo, desplazarse por un área de $3 \mathrm{~m} \times 3 \mathrm{~m}$, o decidir modificar las reglas de una actividad-, pueden hacerse analogías para desempeñarse en otro de lógica similar e incluso superar lo imprevisto.

- La variabilidad sobre temas y condiciones (espacio, lógica, tiempo, relaciones) provoca reinventar, reinvertir lo conocido, lo vivido, lo comprendido, y manejarlo para solucionar la situación original (recordar los mecanismos efectores del movimiento, primera respuesta), para luego resolver y superar una segunda situación (respuesta codificada), donde la movilización de esquemas de acción alimenta (enriquece) una competencia más general y amplia.

- La variabilidad en la práctica no sólo es saber hacer. -Va más allá de la acumulación de conocimientos (declarativos, procedimentales, metamotrices y afectivos), supone el conjunto de disposiciones y esquemas que permiten la movilización de conocimientos en situación, en tiempo útil y de manera adecuada.

Ahora bien, un segundo factor de intervención docente dirigido a la edificación de la competencia motriz es invertir, junto con el trabajo de solución de problemas, la variabilidad de la práctica. Hoy en día, son amplias la difusión y la argumentación pedagógicas que señalan que el procedimiento más adecuado para el despliegue de la motricidad es ofrecer a los escolares experiencias múltiples y variables.

En efecto, una condición básica para el desarrollo de la competencia motriz es la práctica, cargada, como hemos visto, de elementos cognitivos, afectivos y físicos. Las acciones concretas (las prácticas) no equivalen a repetición, mucho menos a automatización. Por el contrario, se hace 
necesario flexibilizar los programas motrices y los patrones de movimiento de tal manera que exista un conjunto de coordinaciones que habiliten a los niños y las niñas a dar respuesta a numerosas y distintas situaciones.

El conjunto de coordinaciones que promueven la práctica variable supone que los niños y adolescentes utilicen sus experiencias previas y la información nueva para:

- Reconocer las diferencias entre lo ya practicado y las realizaciones de la tarea nueva (semejanzas y diferencias).

- Recuperar e integrar experiencias motrices pasadas con las nuevas experiencias perceptivas del cometido presente y percibir el nivel de esfuerzo solicitado.

- Animar la toma de decisiones para realizar los cometidos y tareas.

- Hacer correcciones y una autoobservación en torno a sus realizaciones.

- Ser conscientes de sus desempeños y efectos de sus acciones motrices.

- Poner al día sus esquemas de respuesta y de autorrealización de sus propias percepciones de ser competentes.

La práctica variable implica adaptarse mejor a situaciones nuevas y diferentes, y hacer planes de acción que permitan salir airosos y satisfechos de lo realizado en forma individual. La variabilidad de la práctica se refiere básicamente a la modificación, el conocimiento y la comprensión de los aspectos atinentes a la trayectoria de los objetos e implementos utilizados, a las distintas posiciones y formas de actuar en los espacios y área de juego, a percibir las distancias entre jugadores, a controlar los desplazamientos dentro de los límites de la cancha, a diversificar las formas de manejar los objetos y a darse cuenta de las distintas maneras de desplazarse y actuar en determinada acción.

Variar las condiciones de la práctica significa generar y provocar respuestas motrices diferenciadas que permitan a los estudiantes adaptar sus respuestas y tener plena conciencia y control de sí mismos ante los diversos elementos (velocidad, fuerza, trayectorias, espacio de movilización).

¿Cómo hacer para impulsar una práctica variable dentro de las sesiones de Educación Física? Pueden señalarse cuatro condiciones para que los alumnos logren una mejor disponibilidad corporal ${ }^{2}$, sean competentes

\footnotetext{
${ }^{2}$ El concepto de disponibilidad corporal define la capacidad corporal $y$ mental del organismo para reaccionar globalmente, y sin que medie intelectualización, ante una situación de emergencia en función de sus viintelectualización, ante una situación de
vencias anteriores (Le Boulch, 1991: 60).
}

en sus desempeños y realizaciones, y tomen decisiones. Estas condiciones son: a) el espacio o área de trabajo, b) temporales, c) las interacciones e intercomunicación entre los participantes, y $d$ ) el manejo de los implementos utilizados en las sesiones (Barreiro, 1991, citado por Ruiz, 1995: 75 y ss).

\section{Modificaciones que}

dan paso a la práctica variable

$\mathrm{El}$ área de trabajo circunscribe, en cierto modo, las realizaciones y los desempeños motrices. No es lo mismo desplazarse en un área rectangular de $3 \mathrm{~m} \times 2 \mathrm{~m}$ (espacio correspondiente al tenis de mano), que movilizarse en otra de $10 \mathrm{~m} \times 15 \mathrm{~m}$, o a través de un circuito de acción motriz $z^{3}$ que contenga, por ejemplo, cinco estaciones o bases. En la zona rectangular pequeña los límites del área supeditan la motricidad y "cierran", por así decirlo, los desplazamientos más amplios. En la más grande los desempeños pueden ser, por ejemplo, "correr más rápido", mientras que en el circuito las movilizaciones ofrecen otras posibilidades.

Veamos niveles más detallados de la variabilidad. Nos ubicaremos en la cancha de $3 \mathrm{~m} \times 2 \mathrm{~m}$ y tomaremos el caso del tenis de mano o piso.

\section{Ejemplo 1:}

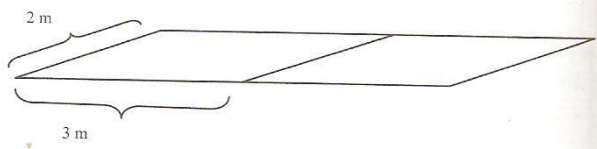

Actividades y características generales de los desempeños motrices:

1. El área que nos corresponde de $3 \mathrm{~m} \times 2 \mathrm{~m}$ la definimos como cancha propia. La acción es individual.

2. El motivo del juego es: devolver la pelota con la palma de la mano, después de botar una sola vez en mi cancha.

3. El set se jugará a 10 tantos.

4. No hay red.

${ }^{3}$ Los circuitos de acción motriz son un recurso didáctico que permite trabajar con pequeños grupos recorriendo en forma simultánea estaciones o bases en una secuencia. Una de sus características pedagógicas consiste en poner a prueba las habilidades psicomotrices y sociomotrices de manera consciente, por lo cual precisan una participación comprometida por par te de los alumnos 


\section{REFLEXIONES ORIGINALES}

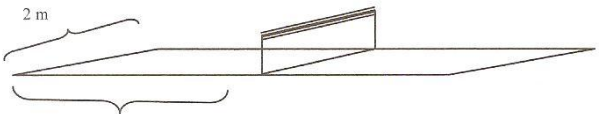

$4 \mathrm{~m}$

Modificaciones a ciertas condiciones que provocan una práctica variable:

1. La cancha propia ahora tendrá $4 \mathrm{~m}$ de profundidad y colocaremos una red a cierta altura. Modificacion del área. La acción continúa individualmente.

2. La pelota podrá botar dos veces en mi cancha. Modificaciones al manejo del implemento, la incertidumbre del bote de la pelota (al hacerlo dos veces), permite que pueda devolverse con más facilidad.

3. El set se disputa a tres minutos. El factor tiempo es el que supedita el logro de los tantos.

Nota. La condición de la intercomunicación e interacción no se modificó; ésta se realizará al momento de hacerlo en dobles.

\section{Ejemplo 2:}

Explorar un patrón de movimiento

Existe la posibilidad didáctica de explorar un patrón de movimiento y dar paso a la variabilidad de la práctica alrededor de él. Tomemos el caso del patrón de movimiento saltar. Al organizar un circuito de acción motriz de cinco bases, tenemos:

\begin{tabular}{|c|c|}
\hline Bases del circulto & Caractensticas de los desempenios \\
\hline Base 1: saltos largos. & $\begin{array}{l}\text { Saltar la mayor distancia con un impulso de } \\
\text { ambas piernas. }\end{array}$ \\
\hline Base 2: saltos a la cuerda. & $\begin{array}{l}\text { Con la cuerda, realizar sattando un recorrido } \\
\text { de ida y vuelta }\end{array}$ \\
\hline Base 3: saltos entre llantas. & $\begin{array}{l}\text { Sattar entre los espacios de las llantas que } \\
\text { están colocadas en hilera. }\end{array}$ \\
\hline $\begin{array}{l}\text { Bese 4: saltos sobre un solo } \\
\text { apoyo. }\end{array}$ & $\begin{array}{l}\text { Saltar sobre un solo pie de ida y de regreso } \\
\text { sobre el otro. }\end{array}$ \\
\hline $\begin{array}{l}\text { Base 5: saltos para tocar } \\
\text { una marca en la pared. }\end{array}$ & $\begin{array}{l}\text { Apoyados en la pared, saltar y tratar de tocar } \\
\text { la marca a cierta aitura. }\end{array}$ \\
\hline $\begin{array}{l}\text { Análisis } \\
\text { Aun cuando el pa } \\
\text { - ¿Son iguales los m } \\
\text { - ¿Son similares los } \\
\text { - ¿Cómo son las caí } \\
\text { - ¿Qué movimiento } \\
\text { ¿Cómo se supera }\end{array}$ & $\begin{array}{l}\text { rón es el mismo: } \\
\text { omentos del despegue del piso? } \\
\text { movimientos en el aire? } \\
\text { das? } \\
\text { s provocan los objetos utilizados? } \\
\text { se objeto? }\end{array}$ \\
\hline
\end{tabular}

La variabilidad puede abrir aun más posibilidades para abundar el tipo de práctica. Las siguientes tablas apuntan en ese sentido:

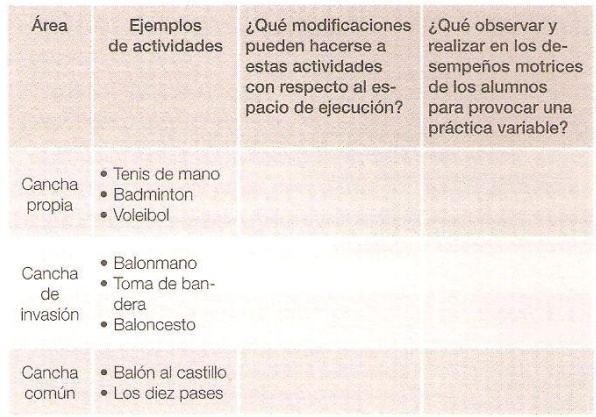

Tabla 1. Condiciones que dan paso a la variabilidad en la práctica, según el espacio o área de trabajo.

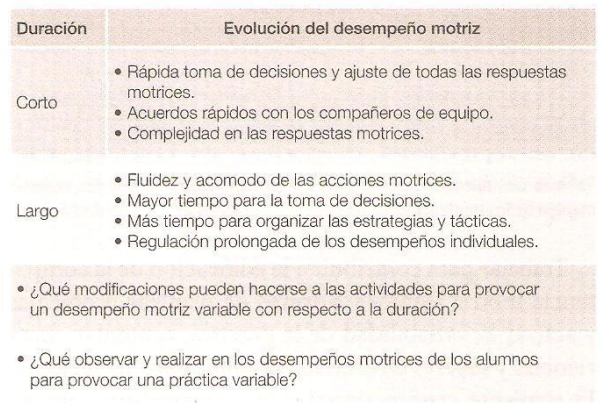

Tabla 2. Condiciones que dan paso a la variabilidad en la práctica, según el tiempo

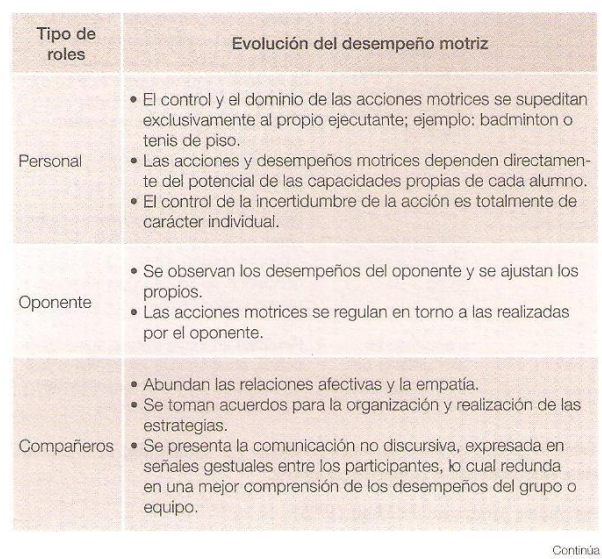




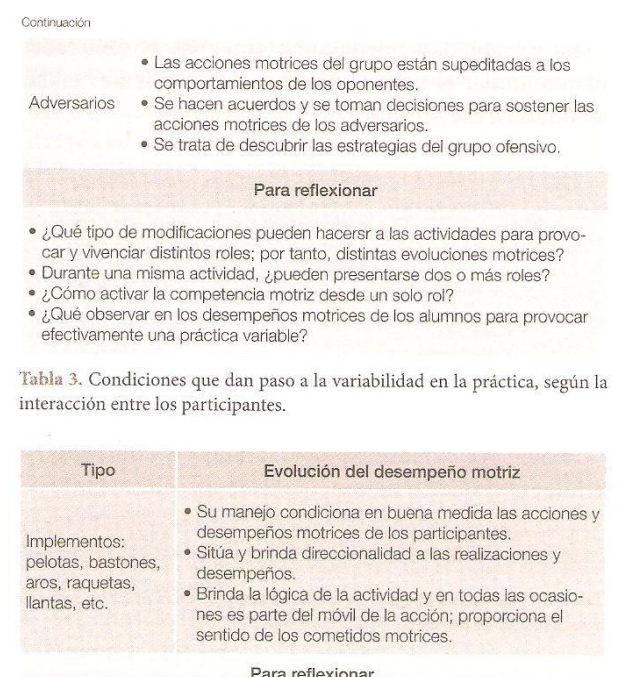

Para reflexionar

- ¿Cómo provocar una práctica variable a partir del manejo de dos implemen-

- ¿Qué sentido le da cierto implemento a los desempeños? ¿Cómo variar? - ¿Que tipo de respuestas obtuvieron los alumnos al modificar el trabajo cen distintos implementos?

Tabla 4. Condiciones que dan paso a la variabilidad en la práctica, según el manejo de los implementos.

Trabajar para contribuir a la edificación de la competencia motriz significa, a través de plantear problemas y activar la variabilidad de la práctica, acumular experiencias y saberes en el conocimiento sobre las acciones. El siguiente cuadro sintetiza las características de ese conocimiento:

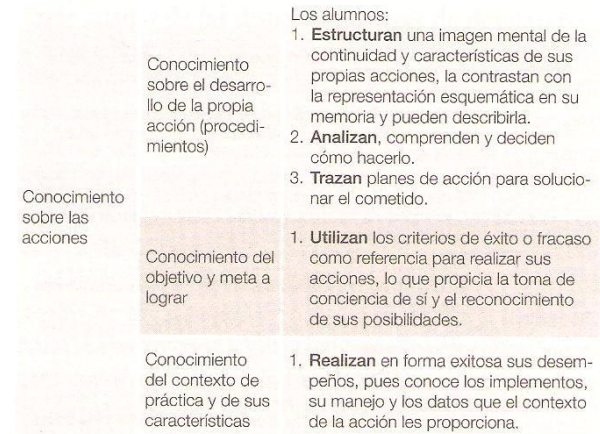

Tabla 5. Efectos de las situaciones-problema y la variabilidad de la práctica en la edificación de la competencia motriz.

Apoyado y parafraseado de Ruiz (1995)
En consecuencia, la intervención docente contribuye a la edificación de la competencia motriz en los niños y niñas al impulsar una abundante y variada práctica; de modo que:

- Debe crear situaciones-problema que movilicen experiencias y saberes orientados a aprendizajes específicos.

- Identifica aprendizajes efectivos y plantea vínculos a partir de la variabilidad de la práctica, entre saber qué se debe hacer y cómo realizarlo.

- Se pone en el lugar de los estudiantes, se comunica con ellos y les ayuda a verbalizar sus acciones.

Éste es precisamente el motivo de la intervención docente que puede desplegarse en las actividades de la Educación Física.

\section{Conclusión}

Después de esta pequeña incursión en torno al saber cómo, y de la contribución que la motricidad y la realización de los desempeños motrices le añaden, conviene reflexionar sobre algunas orientaciones de carácter didáctico que los profesores hemos de tener en cuenta al momento de intervenir didácticamente:

- Comentar a los alumnos el tipo de aprendizajes, desempeños motrices, actitudes, conceptos y procedimientos que han de lograrse al participar y realizar determinadas actividades o juegos. Esto tiene como finalidad provocar la disposición psicológica, motivar la actitud atenta para comprender el tipo de realizaciones, activar la experiencia motriz previa y agilizar el saber cómo, y posibilitar, en conjunto, el cumplimiento de los cometidos y tareas cognitivo-motrices de acuerdo con el principio: es más fácil hacerlo si de antemano conozco lo que hay que realizar. Los recursos pueden ser la verbalización, la demostración, la confección de esquemas y dibujos, la observación de videos, hasta la manipulación de implementos y la modificación de las reglas de juego.

- Escuchar, conocer y tomar en cuenta las propuestas de los alumnos para confeccionar situaciones-problema y ambientes de aprendizaje. La solicitud que las niñas y niños hacen para repetir ciertos juegos y actividades tiene como origen, entre otros: $a$ ) el gusto por reiterar ciertos patrones de movimiento característicos de su edad y desarrollo que les proporcionan satisfacción, confianza y desempeño exitoso; $b$ ) la se- 
guridad en la movilización corporal al participar en un juego o actividad relativamente prevista y conocida, y con ello la presencia de una motricidad significativa anclada necesariamente al saber cómo; y $c$ ) el reconocimiento del logro o meta de esa actividad que se vincula con toda la experiencia previa y con el entramado de respuestas motrices que pueden utilizarse.

- Observar las actitudes de niñas y niños para verificar, en cierta forma, los aprendizajes y conocimientos que están logrando. Una buena manera es comentar la experiencia, qué gustó más y por qué, cuáles fue- ron las dificultades presentadas y cómo pudieron salvarse, cómo fueron los desempeños motrices, cuáles fueron las emociones y sentimientos y cómo se superó en toda la actividad general.

- Insistir y promover la práctica variable. Organizar y presentar rutas de desempeño motriz modificando las condiciones de espacio, tiempo, formas de trabajar con los implementos y abundar en formas de interacción y comunicación entre los participantes.

- Dar espacio para jugar como una forma de actualización del saber cómo, por cuanto éste contiene conocimientos de muy diversa naturaleza.

\section{Referencias}

Aisenstein, A. (coord.). (2000). Repensando la educación física escolar. Entre la educación integral y la competencia motriz. Buenos Aires: Ediciones Novedades Educativas.

Arnold, P. (1988). Educación física, movimiento y curriculum. Madrid: Morata.

Brito Soto, L. F. (2004). El saber cómo: hacer, actuar, desempeñarse, quid del juego y la educación física. Magisterio Educación y Pedagogía, 6, diciembre de 2003-enero de 2004.

Castañer Balcells, M. y Oleguer Camerino, F. (1991). La educación fisica en la enseñanza primaria. Barcelona: Inde.

Famose, J. P. (s. f.). Aprendizaje motor y dificultad de la tarea. Barcelona: Paidotribo.

Le Boulch, J. (1991). El deporte educativo. Psicocinética y aprendizaje motor. Barcelona: Paidós.
Navarro Adelantado, V. (2002). El afán de jugar. Teoría y práctica de los juegos motores. Barcelona: Inde.

Oña Sicila, A. (coord.) (1999). Control y aprendizaje motor. Madrid: Sintesis.

Perrenoud, P. (1999). Construir competencias en la escuela. Santiago: Dolmen Ediciones.

Ruiz Pérez, L. M. (1995). Competencia motriz. Elementos para comprender el aprendizaje motor en educación fisica escolar. Madrid: Gymnos.

Sánchez Bañuelos, F. (coord.) (2003). Didáctica de la educación física. Madrid: Prentice Hall 